\title{
Bilateral peritonsillar abscesses complicating acute tonsillitis
}

\section{Yuan-Yung Lin MD, Jih-Chin Lee MD}

Competing interests: None declared.

This article has been peer reviewed.

Correspondence to: Dr. Jih-Chin Lee, doc30450@gmail.com

CMAJ 2011. DOI:10.1503 /cmaj.100066

A 24-year-old woman presented to the emergency department with a three-day history of worsening sore throat, pain with swallowing and fever. She had been seen by her primary care physician one day before presentation because of sore throat and fever, and had been given oral amoxicillin. She was previously well with no history of recurrent tonsillitis, previous peritonsillar abscess or drug allergies.

On physical examination, her temperature was $39.8^{\circ} \mathrm{C}$, pulse rate was 90 beats $/ \mathrm{min}$, respiratory rate was 24 breaths/min and blood pressure was $110 / 70 \mathrm{~mm} \mathrm{Hg}$. She spoke with a muffled voice without substantial stridor or respiratory distress. Examination of the oral cavity and oropharynx showed moderate trismus, pooling of saliva, symmetrically enlarged and inflamed tonsils, and a bilaterally congested and bulging soft palate with a midline uvula (Figure 1). There was also bilateral, tender submandibular lymphadenopathy. The remainder of the physical examination was unremarkable.

A complete blood count showed a leukocyte count of 17.6 (normal $4.5-11.0) \times 10^{\circ} / \mathrm{L}$ with an elevated absolute neutrophil count of 15.7 (normal $1.8-8.1) \times 10^{9} / \mathrm{L}$. The absolute lymphocyte count and monocyte count were within normal limits. The level of C-reactive protein was 3926.8 (normal < 47.6) nmol/L. A monospot test was not done. On her arrival at the emergency department, the patient was given intravenous (IV) fluids and IV amoxicillin-clavulanic acid for a provisional diagnosis of peritonsillar abscess.

Contrast-enhanced computed tomography

\section{- Ker POINTS}

- Peritonsillar abscess is a potentially life-threatening deep-neck infection in adults that may complicate acute tonsillitis.

- Bilateral swelling of the soft palate with a midline uvula may indicate the presence of bilateral peritonsillar abscesses.

- Contrast-enhanced computed tomography should be considered for assessment of patients with signs and symptoms suggestive of bilateral peritonsillar abscesses.

- A patient with suspected bilateral peritonsillar abscesses requires urgent otolaryngologic assessment.

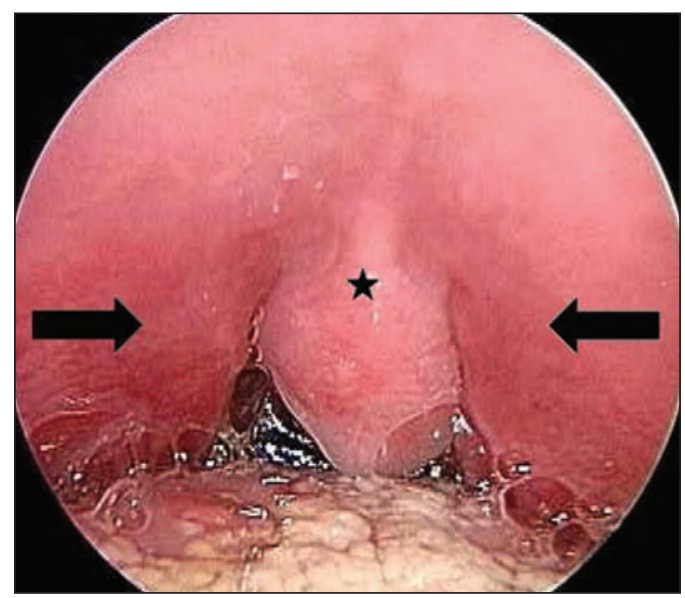

Figure 1: Oral cavity and oropharynx of a 24-yearold woman with worsening sore throat, odynophagia and fever, showing pooling of saliva and a bilaterally congested and swollen soft palate (arrows) with a midline uvula (asterisk).

(CT) of the neck showed bilateral hypodense masses with thick rim enhancement in the superior poles of the peritonsillar regions measuring $2.6 \times 1.8 \mathrm{~cm}$ and $0.8 \times 0.8 \mathrm{~cm}$, respectively (Figure $2 \mathrm{~A}$ ), and extending down to the peritonsillar regions with a multilocular appearance (Figure 2B), consistent with bilateral peritonsillar abscesses. The patient underwent bilateral needle aspiration by the otolaryngologist; a total of 10 $\mathrm{mL}$ of purulent material was obtained from the left side and $3 \mathrm{~mL}$ from the right side. Cultures from the aspiration ultimately grew Acinetobacter baumannii, which was resistant to ampicillin and susceptible to ampicillin-sulbactam. She was discharged home on another course of oral amoxicillin-clavulanic acid for a total of 14 days of antibiotic treatment. The patient's abscesses resolved and there were no signs of recurrence at six months.

\section{Discussion}

Although acute tonsillitis usually runs a relatively benign course, complications may occur (Box 1). ${ }^{1-4}$ These can be life-threatening if treatment is delayed or inadequate. In particular, air- 
way compromise can occur from epiglottis or laryngeal edema; deep-neck abscess of peritonsillar, parapharyngeal or retropharyngeal spaces; and mediastinitis. Other complications related to the sequela of inflammation, septicemia or an untreated group A $\beta$-hemolytic streptococcal pharyngeal infection also can cause high morbidity and mortality.

\section{Epidemiology of peritonsillar abscess}

Peritonsillar abscess is a collection of pus within the space between the tonsil and the superior constrictor muscle. Most instances of peritonsillar abscess are polymicrobial, with a mix of aerobic and anaerobic organisms. The most common aerobes are Streptococcus pyogenes and Streptococcus viridans, whereas Fusobacterium and Bacteroides are among the most common anaerobes. ${ }^{5,6}$ Peritonsillar abscess typically occurs in adolescents and young adults. ${ }^{5}$ It is one of the most common complications of acute tonsillitis; there are about 45000 instances annually in the United States and Puerto Rico. ${ }^{7}$ It is usually unilateral, and clinically evident bilateral presentation is uncommon. The actual frequency of bilateral peritonsillar abscesses is not known; however, it has been seen at rates of $1.9 \%$ to $24 \%$ in reports describing quinsy tonsillectomy (also known as acute abscess tonsillectomy), in which the unsuspected contralateral abscess was discovered during surgery. ${ }^{1,6}$

On reviewing the literature, we found reports on 10 patients (Appendix 1, available at www .cmaj.ca/lookup/suppl/doi:10.1503/cmaj.100066 /-/DC1) with clinical courses similar to that of our patient. Like our patient, these patients had clinically evident bilateral peritonsillar abscesses (in contrast to an unsuspected contralateral peritonsillar abscess found at the time of bilateral quinsy tonsillectomy for a presumed unilateral peritonsillar abscess). The mean age was 22.7 (range 9-32) years. All but one patient had a bilaterally bulging soft palate with a midline uvula on presentation. Six instances were diagnosed by contrast-enhanced CT, two by needle aspiration and two by incision and drainage. Initial management included needle aspiration in four instances, incision and drainage in four, and quinsy tonsillectomy (removal of tonsil to drain abscess) in two. After discharge, five patients underwent elective tonsillectomy, four were followed up (two had previously undergone quinsy tonsillectomy), and the subsequent management was not reported for one patient.

\section{Diagnosis of peritonsillar abscess}

The diagnosis of peritonsillar abscess is usually made on the basis of the history and physical examination. In unilateral or bilateral abscesses, the patient may present with a muffled voice, progressive odynophagia, dysphagia, referred otalgia, trismus, oral pooling of saliva, drooling and fever. ${ }^{1,6,8}$ In unilateral peritonsillar abscess, the classic intraoral finding is bulging of one anterior tonsillar pillar and the adjacent soft palate with contralateral displacement of the uvula. ${ }^{1,2,6,8}$

Patients with bilateral peritonsillar abscesses present a diagnostic dilemma because they do not exhibit the above classic asymmetric signs seen in unilateral peritonsillar abscess, such as asymmetry of the tonsils and palate, and deviation of the uvula. The presence of a midline uvula does not eliminate the possibility of peritonsillar abscess. A midline uvula with bilateral bulging of the soft palate appears to be a key sign in bilateral peritonsillar abscesses, ${ }^{8}$ as was seen in our patient and in case reports in the literature. Although this presentation is uncommon in peritonsillar abscess in general, other features of the physical examination in our patient would have suggested a disease that needed further diagnostic workup. The presence of trismus and a muffled voice are also concerning features that would warrant a thorough workup for worst-case scenarios. ${ }^{1,9}$

\section{Differential diagnosis of bilateral peritonsillar abscesses}

The intraoral examination of severe acute tonsillitis and infectious mononucleosis can be quite similar to that of bilateral peritonsillar abscesses. Other less common conditions may be confused with bilateral peritonsillar abscesses, such as lymphoma presenting with bilateral large and

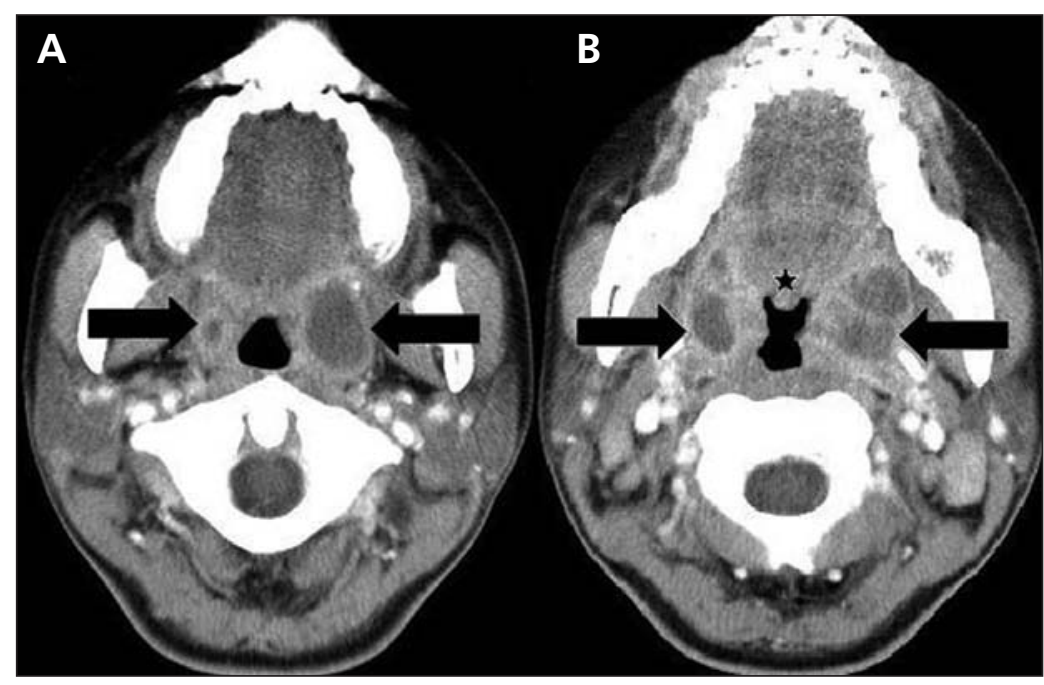

Figure 2: Contrast-enhanced computed tomographic scan of the neck showing a midline uvula (asterisk) and bilateral hypodense masses (arrows) with thick rim enhancement $(A)$ in the superior poles of the peritonsillar regions and (B) extending down to the peritonsillar regions with a multilocular appearance. 
Box 1: Complications of acute tonsillitis ${ }^{1-4}$

- Bilateral or unilateral peritonsillar abscess

- Parapharyngeal abscess

- Retropharyngeal abscess

- Mediastinitis (e.g., descending necrotizing mediastinitis)

- Edema of the epiglottis, larynx or both

- Bacteremia with metastatic seeding, sepsis or both

- Intracranial venous thrombosis

- Carotid artery thrombosis

- Lemierre syndrome (thrombophlebitis of the internal jugular vein)

- Pseudoaneurysm of the internal or external carotid artery

- Nonsuppurative sequelae of group A streptococcal infection (e.g., rheumatic fever, acute glomerulonephritis or guttate psoriasis)

- Inflammatory torticollis, Grisel syndrome or both

bulky tonsils or salivary gland tumours noted in the soft palate. ${ }^{6}$

Contrast-enhanced CT may help to differentiate bilateral peritonsillar abscesses from other diseases and should be considered, not only to confirm the diagnosis, but also to rule out any complications, extension into the deep-neck space or other diseases. Because bilateral peritonsillar abscesses are not common, evidence for this specific diagnosis option is based mainly on case reports. However, the role of CT in diagnosing abscesses in the head and neck region is well established. In light of the proven value of $\mathrm{CT}$ in assessing unilateral peritonsillar abscesses, ${ }^{1,2,6}$ one might conclude that it is reasonable to use the same technology for bilateral peritonsillar abscesses.

\section{Management}

Peritonsillar abscess is a specific deep-neck space infection. It is important to diagnose and treat peritonsillar abscess rapidly and adequately, partly to prevent respiratory obstruction, and partly to avoid perforation of the abscess into the parapharyngeal space with spread along the neck vessels to the mediastinum or skull base. ${ }^{1}$ In managing deep-neck space infection, the first and most important consideration in treating peritonsillar abscess is immediate securing of the airway. Antibiotics should be started, empirically.

Once an abscess has formed, antimicrobial therapy alone may be inadequate and surgical drainage may be necessary. There is no consensus on the ideal surgical procedure for peritonsil- lar abscess, but needle aspiration, incision and drainage, and quinsy tonsillectomy are considered acceptable for the surgical management of acute peritonsillar abscess. An evidence-based review of the treatment of peritonsillar abscess reported that most series found these three approaches were all highly effective for treating unilateral peritonsillar abscess, and the recurrence rates after these procedures are low. ${ }^{5}$ Earlier studies tended to stress the safety of quinsy tonsillectomy and its definitive nature for the treatment of peritonsillar abscess. Later studies emphasized the efficacy of needle aspiration or incision and drainage procedures. ${ }^{5}$ In recent decades, needle aspiration has been favoured because it can be both diagnostic and therapeutic, and provides immediate relief of symptoms with a reported $95 \%$ success rate. ${ }^{5,7}$

In view of the proven efficacy of needle aspiration in treating unilateral peritonsillar abscess, one might conclude that it is reasonable to use the same technique for bilateral peritonsillar abscesses in a controlled situation. Because the risk of complications may be higher with bilateral peritonsillar abscesses than with unilateral disease, a period of close airway observation following needle aspiration is vital. ${ }^{1}$ If there is no improvement, repeat aspiration, incision and drainage, or even tonsillectomy can still be performed.

Quinsy tonsillectomy has been indicated previously for patients not responding to intravenous antibiotic treatment, needle aspiration, and incision and drainage of their peritonsillar abscess. ${ }^{5}$ It also has clinical value for acute management of the severe complications of peritonsillar abscess. If sleep apnea or airway obstruction occur in evident bilateral peritonsillar abscesses, quinsy tonsillectomy should be considered.

Although in our patient cultures ultimately grew A. baumannii, we are not convinced that this was the causative organism. The true microbial cause may have been the more typical mix of streptococci or anaerobes. We chose amoxicillin-clavulanic acid for covering the group A $\beta$-hemolytic streptococci, the most common offending organism; however, we think it was the drainage procedure that was curative.

Bilateral peritonsillar abscesses are uncommon; therefore, it is unlikely a series could be developed that would be amenable to standard investigational analysis of treatment options. Based on our review of case reports, most of the specific treatment options are similar to those used in unilateral peritonsillar abscess. Treatment with combined needle aspiration and antibiotic therapy was successful in our patient, with no further complications. 


\section{References}

1. Fasano CJ, Chudnofsky C, Vanderbeek P. Bilateral peritonsillar abscesses: not your usual sore throat. J Emerg Med 2005; 29:45-7.

2. Rubin MA, Gonzales R, Sande MA. Pharyngitis, sinusitis, otitis, and other upper respiratory tract infections. In: Fauci AS, Braunwald E, Kasper DL, et al., editors. Harrison's principles of internal medicine. 17th ed. New York (NY): McGraw Hill 2008. p. 210-4.

3. Brochu B, Dubois J, Garel L, et al. Complications of ENT infections: pseudoaneurysm of the internal carotid artery. Pediatr Radiol 2004;34:417-20.

4. Galer C, Holbrook E, Treves J, et al. Grisel's syndrome: a case report and review of the literature. Int J Pediatr Otorhinolaryngol 2005;69:1689-92.

5. Johnson RF, Stewart MG, Wright CC. An evidence-based review of the treatment of peritonsillar abscess. Otolaryngol Head Neck Surg 2003;128:332-43.

6. Mobley SR. Bilateral peritonsillar abscess: case report and presentation of its clinical appearance. Ear Nose Throat J 2001; 80:381-2.
7. Herzon FS, Harris P. Mosher Award thesis. Peritonsillar abscess: incidence, current management practices, and a proposal for treatment guidelines. Laryngoscope 1995;105:1-17.

8. Fiechtl JF, Stack LB. Images in clinical medicine. Bilateral peritonsillar abscesses. N Engl J Med 2008;358:e27.

9. Kilty SJ, Gaboury I. Clinical predictors of peritonsillar abscess in adults. J Otolaryngol Head Neck Surg 2008;37:165-8.

Affiliations: From the Department of Otolaryngology Head and Neck Surgery, Tri-Service General Hospital, National Defence Medical Center, Taipei, Taiwan

Contributors: Both authors contributed substantially to the concept, drafting and revising of the article, and both approved the final version submitted for publication.

Acknowledgements: The authors thank Dr. Keng-Kuang Tsai and Dr. Shao-Cheng Liu for their helpful insight and support in writing the article. 